\title{
SYSTEMATICS OF THERMODYNAMIC QUANTITIES IN A 3-REGION PHASE DIAGRAMME FROM STRANGE PARTICLE RATIOS
}

\author{
A.D. Panagiotou \\ CERN, Geneva, Switzerland ${ }^{1)}$ \\ G. Mavromanolakis and J. Tzoulis \\ Department of Physics, University of Athens, Athens, Greece
}

\begin{abstract}
Using the strange-hadron mass spectrum, the $\mu_{\mathrm{q}} / T$ and $\mu_{\mathrm{s}} / T$ values obtained from strange particle ratios, we estimate the temperature of the equilibrated primordial state for several nuclear interactions at AGS and SPS. Fitting the systematics for $T, \mu_{\mathrm{q}}$ and transverse flow velocity we predict their values at RHIC. On the basis of lattice calculations and experimental evidence, we propose the existence of an intermediate phase: the Deconfined Quark Matter (DQM) phase with massive and correlated quarks. We define the $\mu_{\mathrm{s}}=0$ curve as the boundary between the HG and DQM phases for the strange-hadron sector and write an empirical EoS for the latter region. Its distinct characteristic is the negative strange-quark chemical potential. Experimental suggestion for $\mu_{\mathrm{S}}<0$ may have been seen in $\mathrm{S}+\mathrm{A}$ interactions at $200 \mathrm{~A} \mathrm{GeV}$.
\end{abstract}

To appear in Proceedings of "Strangeness '96", May 1996, Budapest

\footnotetext{
1) On leave from the University of Athens
} 
It is generally expected that ultra-relativistic nucleus-nucleus collisions will provide the basis for strong interaction thermodynamics, which will lead to new physics. Accurate knowledge of (thermo)dynamic quantities, such as the temperature and chemical potentials of the primordial state, and the transverse flow velocity, will be invaluable for the identification of new phenomena.

On a phenomenological level, we expect strongly interacting matter to undergo two consecutive phase transitions: quark deconfinement and chiral symmetry restoration. The former takes place earlier and is followed by the diminishing of qq correlation, as the result of colour charge screening, and by the decrease of the (deconfined) quark mass with increasing temperature. It finally leads to full chiral symmetry restoration and the formation of ideal Quark-Gluon Plasma (QGP). The region mapped on the phase diagramme during their effect creates an intermediate region, the Deconfined Quark Matter (DQM) phase, between the Hadron Gas (HG) and QGP phases. We propose such a 3-region phase diagramme and show that it can be described by the variation in the strange-quark chemical potential of the system.

\section{THE FORMALISM}

The strange-hadron mass spectrum is given by the partition function in the Boltzmann approximation. We assume that the system has attained (at least local) thermal and chemical equilibration of three flavours $[1,2]$.

$$
\begin{gathered}
\ln \mathrm{Z}_{\mathrm{HG}}(V, T, \lambda)=\sum_{\mathrm{k}} \mathrm{Z}_{\mathrm{k}} \Pi \lambda_{\mathrm{i}}^{\mathrm{k}} \\
\mathrm{Z}_{\mathrm{k}}(V, T)=\left(V T^{3} / 2 \pi^{2}\right) \sum_{\mathrm{j}} g_{\mathrm{j}}\left(m_{\mathrm{j}} / T\right)^{2} \mathrm{~K}_{2}\left(m_{\mathrm{j}} / T\right),
\end{gathered}
$$

where the fugacity $\lambda_{i}^{\mathrm{k}}$ controls the quark content of the k-strange hadron and $\mathrm{i}=\mathrm{s}$ and $\mathrm{b}$ for the strangeness and baryon number $\left(\lambda_{\mathrm{b}}=\lambda_{\mathrm{q}}^{3}=\exp \left(3 \mu_{\mathrm{q}} / T\right)\right.$, respectively. The summation in (2) indicates the sum of all resonances of each hadron species with mass $m_{\mathrm{j}}$; the degeneracy factor $g_{\mathrm{j}}$ counts the spin and isospin degrees of freedom of the j-resonance. Kaons with mass up to $2045 \mathrm{MeV} / \mathrm{c}^{2}$, hyperons up to $2350 \mathrm{MeV} / \mathrm{c}^{2}$ and cascades up to $2025 \mathrm{MeV} / c^{2}$ are included. We assume isospin symmetry in (1) $\left(\mu_{\mathrm{u}}=\mu_{\mathrm{d}}=\mu_{\mathrm{q}}\right)$. Strangeness neutrality gives:

$$
\left\langle\mathrm{N}_{\mathrm{s}}-\mathrm{N}_{\overline{\mathrm{s}}}\right\rangle=(T / V) \mathrm{d} / \mathrm{d} \mu_{\mathrm{s}}\left[\ln \mathrm{Z}_{\mathrm{HG}}\left(V, T, \lambda_{\mathrm{s}}, \lambda_{\mathrm{q}}\right)\right]=0
$$

which reduces to

$$
\mathrm{Z}_{\mathrm{K}}\left(\lambda_{\mathrm{s}} \lambda_{\mathrm{q}}^{-1}-\lambda_{\mathrm{q}} \lambda_{\mathrm{s}}^{-1}\right)+\mathrm{Z}_{\mathrm{Y}}\left(\lambda_{\mathrm{s}} \lambda_{\mathrm{q}}^{2}-\lambda_{\mathrm{s}}^{-1} \lambda_{\mathrm{q}}^{-2}\right)+2 \mathrm{Z}_{\Xi}\left(\lambda_{\mathrm{s}}^{2} \lambda_{\mathrm{q}}-\lambda_{\mathrm{s}}^{-2} \lambda_{\mathrm{q}}^{-1}\right)=0
$$

Defining the quantity

$$
\gamma=\lambda_{\mathrm{s}} \lambda_{\mathrm{q}}^{-1}=\exp \left(\mu_{\mathrm{s}} / T\right) \exp \left(-\mu_{\mathrm{q}} / T\right)
$$

(4a) becomes

$$
2 \mathrm{Z}_{\Xi} \lambda_{\mathrm{q}}^{6} \gamma^{4}+\lambda_{\mathrm{q}}^{3}\left(\mathrm{Z}_{\mathrm{K}}+\mathrm{Z}_{\mathrm{Y}} \lambda_{\mathrm{q}}^{3}\right) \gamma^{3}-\left(\mathrm{Z}_{\mathrm{Y}}+\mathrm{Z}_{\mathrm{K}} \lambda_{\mathrm{q}}^{3}\right) \gamma-2 \mathrm{Z}_{\Xi}=0
$$


Equation (4) defines the strange-quark chemical potential in terms of $\mu_{\mathrm{q}}$ and $T$. It interrelates the three thermodynamic quantities, $\mu_{\mathrm{s}}, \mu_{\mathrm{q}}$ and $T$, in the equilibrated primordial state $^{1)}$. We use this relation to determine the temperature of the state, knowing the values of $\mu_{\mathrm{s}} / T$ and $\mu_{\mathrm{q}} / T$ from experimental strange-particle ratios [2]. It is simply written as

$$
\mu_{\mathrm{s}}^{\mathrm{HG}}\left(T, \mu_{\mathrm{q}}\right)=\mu_{\mathrm{q}}+T \ln \gamma \quad \gamma=\mathrm{f}\left(T, m_{\mathrm{k}}\right),
$$

$\gamma$ is calculated from $(4 \mathrm{c})$.

In the HG phase with $\mu_{\mathrm{q}}>0$, the coupling of $\mu_{\mathrm{q}}$ and $\mu_{\mathrm{s}}$ in strange hadrons does not require that $\mu_{\mathrm{s}}=0$ everywhere in this phase. The condition $\mu_{\mathrm{s}}=0$ requires that $\lambda_{\mathrm{s}}=\lambda_{\mathrm{s}}^{-1}=1$, and (4a) becomes [3]

$$
\left[\mathrm{Z}_{\mathrm{Y}}\left(\lambda_{\mathrm{q}}+\lambda_{\mathrm{q}}^{-1}\right)-\mathrm{Z}_{\mathrm{K}}+2 \mathrm{Z}_{\Xi}\right]\left(\lambda_{\mathrm{q}}-\lambda_{\mathrm{q}}^{-1}\right)=0
$$

The first factor gives $\mu_{\mathrm{q}}$ as a function of $T$, for which $\mu_{\mathrm{s}}=0$ :

$$
\mu_{\mathrm{q}}=T \cosh ^{-1}\left(\mathrm{Z}_{\mathrm{K}} / 2 \mathrm{Z}_{\mathrm{Y}}-\mathrm{Z}_{\Xi} / \mathrm{Z}_{\mathrm{Y}}\right) .
$$

Note that the vanishing of the strange-quark chemical potential on this line has nothing to do with the QGP phase, where $\mu_{\mathrm{s}}=0$ by definition.

\section{ANALYSIS OF STRANGE-PARTICLE DATA}

\section{$3.1 \quad$ Temperature}

Using Eq. (5), we analysed several nucleus-nucleus interactions from AGS and SPS. Table 1 lists the thermodynamic quantities of the primordial state for all systems considered. Intuitively, the maximum temperature of the state should depend on the total energy in the c.m. system divided by the number of participant nucleons sharing this energy: $T_{\mathrm{o}} \propto \sqrt{\mathrm{s}} /$ participant, where the number of participants is $\mathrm{A}_{\mathrm{part}}=\mathrm{A}_{\mathrm{pr}}\left[1+1.4\left(\mathrm{~A}_{\mathrm{tg}} / \mathrm{A}_{\mathrm{pr}}\right)^{1 / 3}\right]$. This is shown in Fig. 1 for mid-rapidity data of central interactions: $\mathrm{Si}+\mathrm{Au}$ (AGS) and $\mathrm{S}+\mathrm{A}$ (SPS). We also include the temperature of $\mathrm{Au}+\mathrm{Au}(1.15 \mathrm{~A} \mathrm{GeV})$ [4]. Predictions are summarized in Table 1.

For $\mathrm{Au}+\mathrm{Au}$ interactions we studied the variation of the temperature $T_{\mathrm{o}}$ as a function of the total c.m. energy (Fig. 2a). The two lower points are experimental [4, 5], whilst the others are the predictions (Table 1).

\subsection{Transverse flow}

The compression of nuclear fluid at the initial stage of the collision results in a radial flow of the participant matter. One can determine the flow velocity, $\beta=\mathrm{v} / \mathrm{c}$, if one knows the temperature $T_{\mathrm{o}}$ and the inverse slope parameter I, using the Doppler shift formula which interrelates the two quantities:

$$
T_{\mathrm{o}}=\mathrm{I} \sqrt{ }[(1-\beta) /(1+\beta)] \rightarrow \beta=\left[1-\left(T_{\mathrm{o}} / \mathrm{I}\right)^{2}\right] /\left[1+\left(T_{\mathrm{o}} / \mathrm{I}\right)^{2}\right]
$$

\footnotetext{
1) In central nuclear collisions, the mid-rapidity (equilibrated) primordial state is characterized by the thermodynamic quantities: $\mu_{\mathrm{b}}, \mu_{\mathrm{s}}, T, \mathrm{~S}_{\mathrm{b}}\left(T, \mu_{\mathrm{b}}\right)$. The baryo-chemical potential in the primordial state is characteristic of the interacting system. It is conserved and survives through hadronization. The entropy (per baryon) is also conserved, assuming isentropic hadronization. Since $S_{b}$ is a function of $T$ and $\mu_{\mathrm{b}}$, it follows that the temperature of the state should be conserved and survive through hadronization. This leads to the conservation of the strange-quark chemical potential, since the quantities $\mu_{\mathrm{b}}, \mu_{\mathrm{s}}$ and $T$ are related through Eq. (5), which refers to the primordial state. Therefore, the conservation (and survival through hadronization) of $\mu_{\mathrm{b}}, \mu_{\mathrm{s}}$ and $T$ of the state, together with their appropriate inter-relation, allow us to determine the primordial values of these quantities through the experimental strange-particle ratios, which give $\left(\mu_{\mathrm{b}} / T\right)$ and $\left(\mu_{\mathrm{s}} / T\right)$.
} 
Table 1: Thermodynamic quantities obtained from experiments and from our analysis. The $(\diamond)$ denotes predictions based on the current AGS and SPS data.

\begin{tabular}{lccc}
\hline Interaction & $T(\mathrm{MeV})$ & $\mu_{\mathrm{q}}(\mathrm{MeV})$ & $\beta$ \\
\hline $\mathrm{BEVELAC}$ & & & \\
$\mathrm{Au}+\mathrm{Au}, 0.15 \mathrm{~A} \mathrm{GeV}$ & $32 \pm 10$ & & $0.12 \pm 0.05$ \\
$\mathrm{Au}+\mathrm{Au}, 1.15 \mathrm{~A} \mathrm{GeV}$ & $81 \pm 24$ & $158 \pm 25$ & $0.32 \pm 0.05$ \\
$\mathrm{AGS}$ & & & \\
$\mathrm{Si}+\mathrm{Au}, 14.5 \mathrm{~A} \mathrm{GeV}$ & $129 \pm 5$ & $170 \pm 10$ & $0.39 \pm 0.04$ \\
$\diamond \mathrm{Au}+\mathrm{Au}, 10.5 \mathrm{~A} \mathrm{GeV}$ & $126 \pm 9$ & $180 \pm 22$ & $0.38 \pm 0.08$ \\
$\mathrm{CERN}$ & & & \\
$\mathrm{S}+\mathrm{W}(\mathrm{WA} 85) 200 \mathrm{~A} \mathrm{GeV}$ & $197 \pm 7$ & $83 \pm 7$ & $0.17 \pm 0.06$ \\
$\mathrm{~S}+\mathrm{Ag}(\mathrm{NA} 35) 200 \mathrm{~A} \mathrm{GeV}$ & $182 \pm 19$ & $88 \pm 12$ & $0.23 \pm 0.11$ \\
$\mathrm{~S}+\mathrm{S}(\mathrm{NA} 35) 200 \mathrm{~A} \mathrm{GeV}$ & $183 \pm 15$ & $71 \pm 10$ & $0.10 \pm 0.09$ \\
$\mathrm{~S}+\mathrm{S}(\mathrm{WA} 94) 200 \mathrm{~A} \mathrm{GeV}$ & $200 \pm 4$ & $80 \pm 4$ & $0.05 \pm 0.03$ \\
$\diamond \mathrm{Pb}+\mathrm{Pb}, 158 \mathrm{~A} \mathrm{GeV}$ & $194 \pm 13$ & $142 \pm 18$ & $0.27 \pm 0.07$ \\
$\diamond \mathrm{Pb}+\mathrm{Pb}, 80 \mathrm{~A} \mathrm{GeV}$ & $176 \pm 11$ & $152 \pm 19$ & $0.30 \pm 0.07$ \\
$\diamond \mathrm{Pb}+\mathrm{Pb}, 40 \mathrm{~A} \mathrm{GeV}$ & $159 \pm 11$ & $162 \pm 20$ & $0.33 \pm 0.07$ \\
$\diamond \mathrm{Ag}+\mathrm{Ag}, 200 \mathrm{~A} \mathrm{GeV}$ & $200 \pm 13$ & $117 \pm 15$ & $0.20 \pm 0.06$ \\
$\mathrm{RHIC}$ & & & \\
$\diamond \mathrm{Au}+\mathrm{Au}, \sqrt{\mathrm{s}}=200 \mathrm{~A} \mathrm{GeV}$ & $322 \pm 22$ & $64 \pm 11$ & $0.05 \pm 0.05$ \\
$\diamond \mathrm{Au}+\mathrm{Au}, \sqrt{\mathrm{s}}=100 \mathrm{~A} \mathrm{GeV}$ & $286 \pm 19$ & $87 \pm 12$ & $0.11 \pm 0.05$ \\
$\diamond \mathrm{Au}+\mathrm{Au}, \sqrt{\mathrm{s}}=50 \mathrm{~A} \mathrm{GeV}$ & $249 \pm 16$ & $107 \pm 14$ & $0.17 \pm 0.06$ \\
$\diamond \mathrm{Ni}+\mathrm{Ni}, \sqrt{\mathrm{s}}=200 \mathrm{~A} \mathrm{GeV}$ & $322 \pm 22$ & $25 \pm 9$ & 0.0 \\
\hline
\end{tabular}

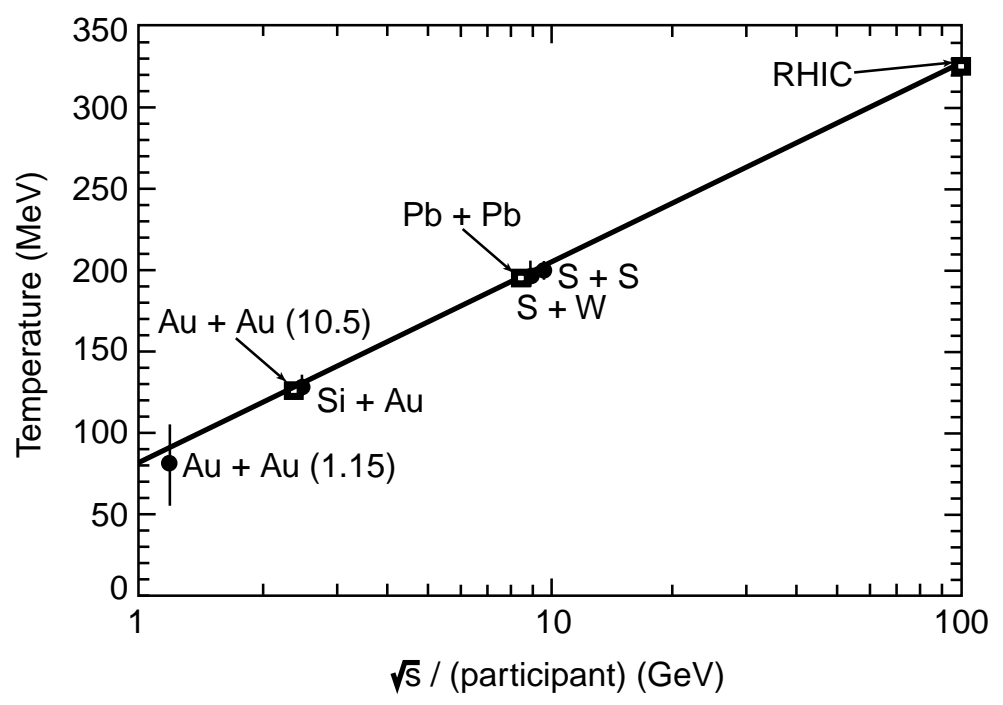

Figure 1: Temperature vs. $(\sqrt{s} /$ participant). Small squares indicate predictions for corresponding interactions. 
Intuitively, we anticipate the compression of nuclear matter (and correspondingly the transverse flow) to be inversely proportional to the total energy per participant nucleon in the centre-of-mass system, divided by the number of participants:

$$
\beta \propto\left[\sqrt{\mathrm{s}} /(\text { participant })^{2}\right]^{-1} .
$$

The results are summarized in Table 1.

The transverse flow velocity is expected to increase with increasing incident energy up to the point where nuclear transparency sets in. Increasing the energy, both compression and transverse flow should decrease and become null at the energy where full nuclear transparency is established. Note that this is a combined effect of the incident energy and the participant mass of the colliding system.

In Fig. $2 \mathrm{~b}$ we plot the $\mathrm{Au}+\mathrm{Au}(1.15 \mathrm{~A} \mathrm{GeV}, 0.15 \mathrm{~A} \mathrm{GeV})$ data and the predicted values for $10.5 \mathrm{~A} \mathrm{GeV}$ and $\sqrt{\mathrm{s}}=50,100$ and $200 \mathrm{~A} \mathrm{GeV}$. It suggests that the disappearance of transverse flow at low energy occurs at $\sqrt{\mathrm{s}} \sim 1.35 \mathrm{~A} \mathrm{GeV}$, in accordance with other experimental evidence [6]. The disappearance of flow at high energy is indicated at $\sqrt{\mathrm{s}}>$ $200 \mathrm{~A} \mathrm{GeV}$. The maximum $\beta$, of the order of 0.4 , is observed at $\sqrt{\mathrm{s}} \sim(4-5) \mathrm{A} \mathrm{GeV}$ for the $\mathrm{Au}+\mathrm{Au}$ system. Note, the flow estimated using $T_{\mathrm{o}}$ is smaller than that estimated using the (lower) freeze-out temperature.

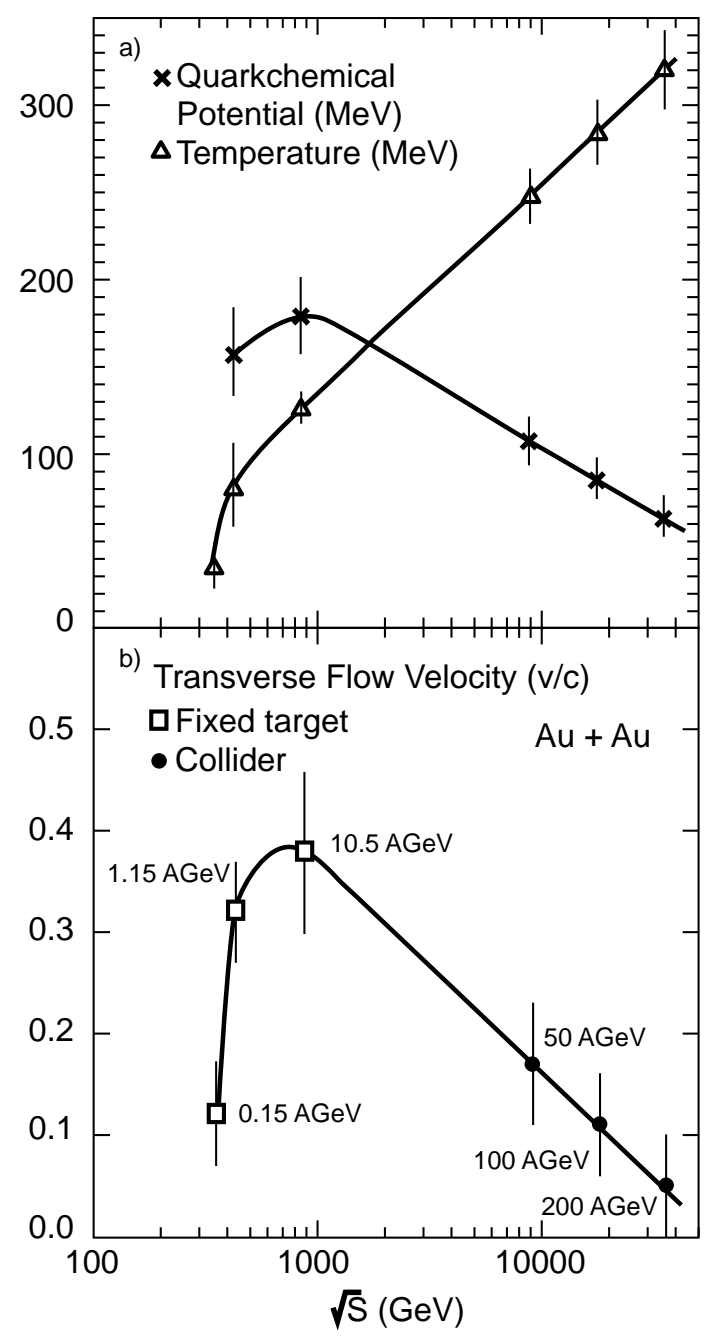

Figure 2: (a) Quarkchemical potential and temperature vs. $\sqrt{s}$ for $\mathrm{Au}+\mathrm{Au}$; (b) transverse flow velocity. Lines are to guide the eye. 


\subsection{Quarkchemical potential}

The quarkchemical potential in a nuclear matter state represents the baryon number concentration. This in turn depends on the matter density. We anticipate, therefore, the quarkchemical potential to be inversely proportional to the total energy per participant in the c.m. system, divided by the number of participant nucleons: $\mu_{\mathrm{q}} \propto$ $\left[\sqrt{\mathrm{s}} /(\text { participant })^{2}\right]^{-1}$. The results of our estimations are listed in Table 1 . In Fig. 2a we show the systematics of the quarkchemical potential for $\mathrm{Au}+\mathrm{Au}$ as a function of $\sqrt{\mathrm{s}}$. The maximum value of $\mathrm{O}(200 \mathrm{MeV})$ is achieved at $\sqrt{\mathrm{s}} \sim(4-5) \mathrm{A} \mathrm{GeV}$. The baryon density, which is a function of $\mu_{\mathrm{q}}$ and $T$, peaks at higher $\sqrt{\mathrm{s}}$, as seen in Fig. 3 for the $\mathrm{Pb}+\mathrm{Pb}$ system.

Figure 4 shows the phase diagramme and the location of the states produced in nuclear interactions in ongoing and future experiments.

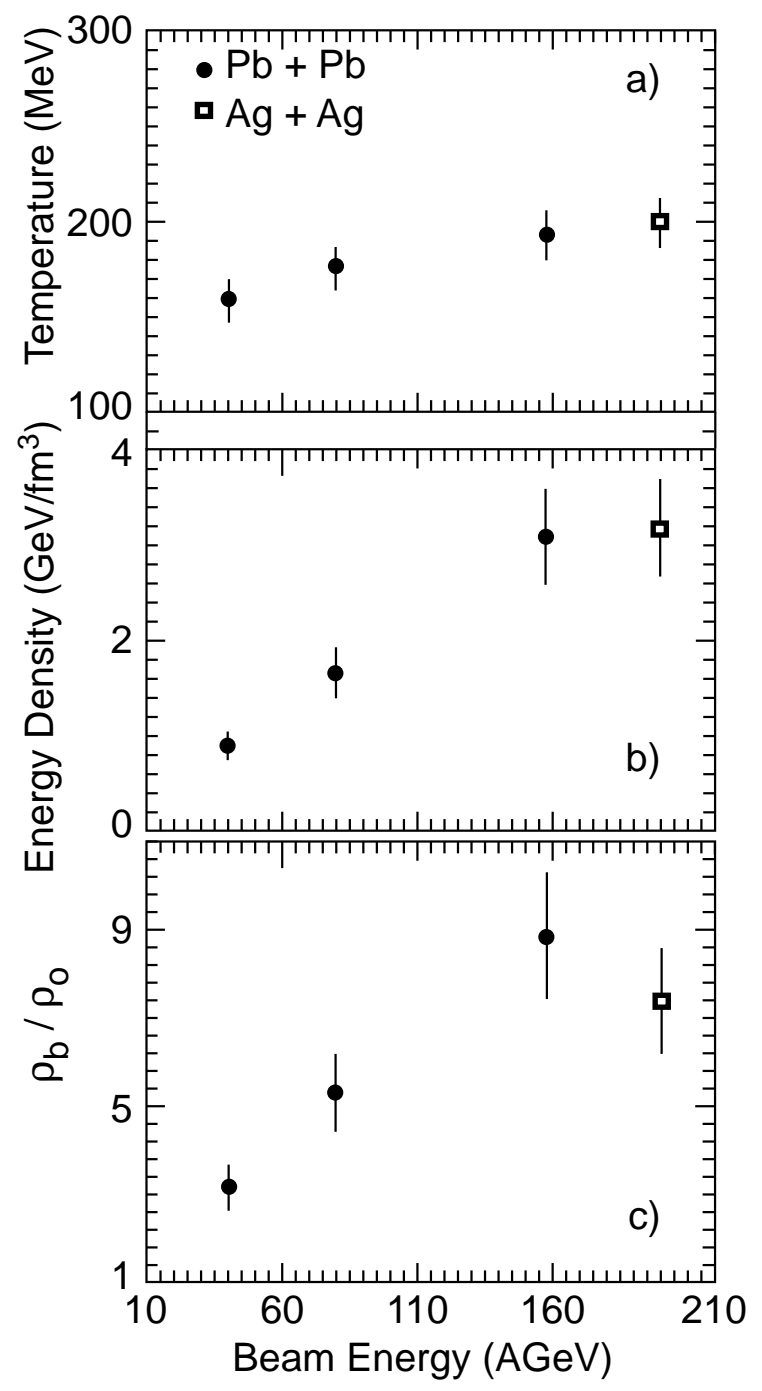

Figure 3: Thermodynamic quantities for $\mathrm{Pb}+\mathrm{Pb}$ and $\mathrm{Ag}+\mathrm{Ag}$ at several energies. 


\subsection{DQM phase}

We consider the region between the two curves to be the Deconfined Quark Matter phase with massive and correlated quarks. In the DQM phase the bag pressure has vanished and $\Delta \varepsilon$ (physical-pertubative) $=0$. Near the HG boundary, quarks have near constituent mass and the correlation between qq is maximum, a prelude to confinement into hadrons. The correlation of the deconfined quarks diminishes as colour charge density increases with temperature. Similarly, the mass decreases at the DQM $\rightarrow$ QGP transition (full chiral symmetry restoration) and quarks attain current masses.

The necessity for a DQM phase is born out of lattice calculations of the order parameters, which even for vanishing baryon number are not 'step functions', but have finite width. For $\mu_{\mathrm{b}}>0$ and/or the inclusion of the strange quark, this is much more evident [9a,b]. This is also alluded to experimentally by $\mathrm{S}+\mathrm{A}$ interactions at $200 \mathrm{~A} \mathrm{GeV}$ [2], where $\left\langle\mu_{\mathrm{S}}\right\rangle \sim 0 \mathrm{MeV}$ and $\varepsilon_{\mathrm{HG}} \sim \varepsilon_{\mathrm{QGP}}\left(a_{\mathrm{s}} \sim 0.6\right)$ for 3-quark flavours, indicating the vanishing difference of the energy density between true and perturbative vacua on the $\mu_{\mathrm{s}}=0$ curve. It also suggests the necessity for an intermediate DQM phase, since interactions should not go directly from HG $\left(a_{s} \sim 1\right)$ to ideal QGP $\left(a_{\mathrm{s}} \sim 0\right)$, but through a transition region where $1>a_{\mathrm{s}}>0$.

In constructing the EoS in the DQM phase, we use the two order parameters: the average thermal Wilson loop, $\langle\mathrm{L}\rangle=\mathrm{R}_{\mathrm{d}}\left(T, \mu_{\mathrm{q}}\right) \sim 0 \rightarrow 1$, as $T=T_{\mathrm{d}} \rightarrow T_{\mathrm{qgp}}$, describing the quark deconfinement-uncorrelation, and the scalar quark density, $\langle\bar{\psi} \psi\rangle=\mathrm{R}_{\mathrm{ch}}\left(T, \mu_{\mathrm{q}}\right) \sim$ $1 \rightarrow 0$, as $T=T_{\mathrm{d}} \rightarrow T_{\mathrm{qgp}}$, denoting the scaling of the quark mass. Even though this state is above $T_{\mathrm{d}}$, the deconfined quarks remain correlated as 'hadron-like states' for a substantial interval. The diminishing of this correlation, as a result of the increase of colour charge screening, is approximated by the factor $\left(1-\mathrm{R}_{\mathrm{d}}\right) \sim 1 \rightarrow 0$, as $T=T_{\mathrm{d}} \rightarrow T_{\mathrm{qgp}}$.

The mass of the quarks decreases after $T_{\mathrm{d}}$ and reaches the bare quark value $\left(m_{\mathrm{u}} \sim\right.$ $\left.5 \mathrm{MeV}, m_{\mathrm{d}} \sim 9 \mathrm{MeV}, m_{\mathrm{s}} \sim 170 \mathrm{MeV}\right)$ at $T_{\mathrm{qgp}}$. To approximate this and also to account for the 'effective mass' of the state, we use the scaled partition function of the HG, ln $\mathrm{Z}_{\mathrm{HG}}{ }^{*}$, where the strange hadron mass $m_{\mathrm{k}}{ }^{*}$, decreases with increasing temperature and assumes, at $T_{\mathrm{qgp}}$, the minimum value, $m_{\mathrm{k}}{ }^{\circ}$, equal to the sum of its bare quark masses: $m_{\mathrm{K}}{ }^{\mathrm{o}} \sim 175 \mathrm{MeV}, m_{\mathrm{Y}}{ }^{\mathrm{o}} \sim 185 \mathrm{MeV}, m_{\mathrm{X}}{ }^{\mathrm{o}} \sim 350 \mathrm{MeV}$. The hadron masses scale according to: $m_{\mathrm{k}}{ }^{*}=\mathrm{R}_{\mathrm{ch}}\left[m_{\mathrm{k}}-m_{\mathrm{k}}{ }^{\mathrm{o}}\right]+m_{\mathrm{k}}{ }^{\mathrm{o}}$.

The gluon field, producing independent $q \bar{q}$ pairs with current masses, contributes to the formation of the QGP state at the rate of deconfinement $R_{d}$. Employing the described dynamics in the DQM phase, we construct the empirical EoS:

$$
\ln \mathrm{Z}_{\mathrm{DQM}}(V, T, \lambda) \sim\left[1-\mathrm{R}_{\mathrm{d}}\left(T, \mu_{\mathrm{q}}\right)\right] \ln \mathrm{Z}_{\mathrm{HG}}{ }^{*}(V, T, \lambda)+\mathrm{R}_{\mathrm{d}}\left(T, \mu_{\mathrm{q}}\right) \ln \mathrm{Z}_{\mathrm{QGP}}(V, T, \lambda) .
$$

Strangeness neutrality gives

$$
\mu_{\mathrm{s}}^{\mathrm{DQM}}\left(T, \mu_{\mathrm{q}}\right)=\mu_{\mathrm{q}}+T \ln \gamma, \quad \gamma=\mathrm{f}\left(T, m_{\mathrm{k}}{ }^{*}\right) .
$$

$\gamma$ now includes the $\left(T, \mu_{\mathrm{q}}\right)$-dependent factors $\mathrm{R}_{\mathrm{ch}}$ and $\mathrm{R}_{\mathrm{d}}$, as well as the EoS in the QGP phase. It is given by [compare with $(4 \mathrm{c})]$

$$
\begin{aligned}
\left(1-\mathrm{R}_{\mathrm{d}}\right) 2 \mathrm{Z}_{\Xi}{ }^{*} \lambda_{\mathrm{q}}^{6} \gamma^{4}+ & {\left[\left(1-\mathrm{R}_{\mathrm{d}}\right)\left(\mathrm{Z}_{\mathrm{K}}{ }^{*}+\mathrm{Z}_{\mathrm{Y}}{ }^{*} \lambda_{q}^{3}\right) \lambda_{q}^{3}+\mathrm{R}_{\mathrm{d}}\left(3 V / \pi^{2}\right)\left(m_{\mathrm{s}} / T\right)^{2} T^{3} \mathrm{~K}_{2}\left(m_{\mathrm{s}} / T\right) \lambda_{\mathrm{q}}^{4}\right] \gamma^{3} } \\
- & {\left[\left(1-\mathrm{R}_{\mathrm{d}}\right)\left(\mathrm{Z}_{\mathrm{Y}}{ }^{*}+\mathrm{Z}_{\mathrm{K}}{ }^{*} \lambda_{\mathrm{q}}^{3}\right)+\mathrm{R}_{\mathrm{d}}\left(3 V / \pi^{2}\right)\left(m_{\mathrm{s}} / T\right)^{2} T^{3} \mathrm{~K}_{2}\left(m_{\mathrm{s}} / T \lambda_{\mathrm{q}}^{2}\right] \gamma\right.} \\
- & \left(1-\mathrm{R}_{\mathrm{d}}\right) 2 \mathrm{Z}_{\Xi}{ }^{*}=0
\end{aligned}
$$

Figure 5 shows the variation of $\mu_{\mathrm{s}}{ }^{\mathrm{DQM}}(T)$ (solid line) for $\mu_{\mathrm{q}}=0.4 T$. We also show the variation of $\mu_{\mathrm{S}}{ }^{\mathrm{DQM}}$ with only the term $\ln \mathrm{Z}_{\mathrm{HG}}{ }^{*}$ in Eq. (10) and the behaviour of Eq. (5) for comparison. 


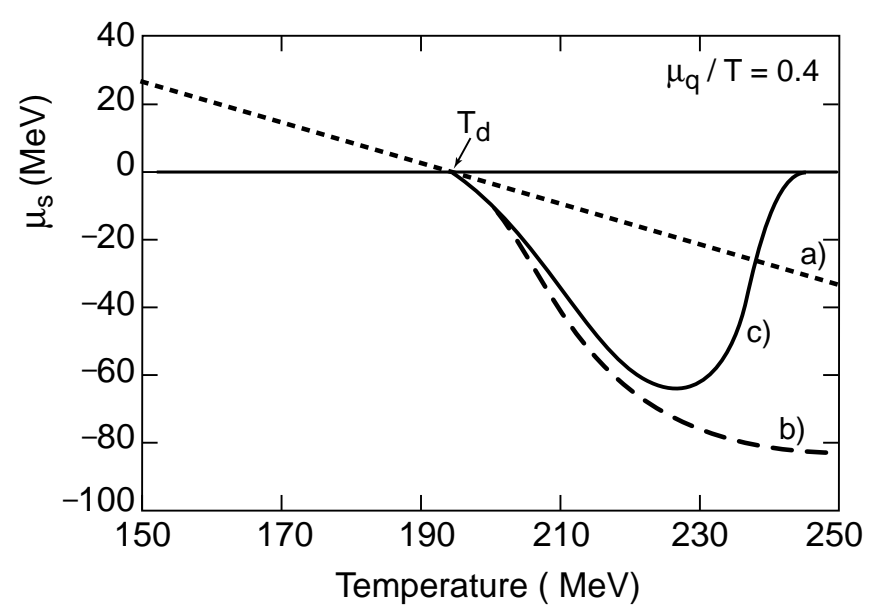

Figure 5: $\mu_{\mathrm{s}}$ vs. $T$ in DQM phase (see text).

Note that:

a) Equation (5) gives small, monotonically negative values for $\mu_{\mathrm{s}}$ in this region, continuing from the HG phase (dotted curve). However, pure hadronic states are not realistic or viable at such high temperatures.

b) Scaling alone of the hadron (quark) masses produces abruptly large negative strange quarkchemical potential, saturating at high temperatures without ever reaching the QGP phase value of zero (dashed curve).

c) Equation (11) gives large negative values for $\mu_{\mathrm{s}}$, which, after reaching a minimum, returns to zero as the QGP phase is approached. The upturn of the curve is caused by the diminishing of the mass-scaled 'hadron-like states' (vanishing of qq correlation) and by the increasing contribution of the QGP state with temperature.

\section{STRANGE-QUARK CHEMICAL POTENTIAL}

Figure 6 shows the variation of the strange-quark chemical potential, throughout the phase diagramme, on the vertical plane formed by the $\mu_{\mathrm{s}}$-axis and the line $\mu_{\mathrm{q}}=0.4 T$ on the $\left(T-\mu_{\mathrm{q}}\right)$-plane. $\mu_{\mathrm{s}}$ attains positive values in the HG because of the coupling between $\mu_{\mathrm{q}}$ and $\mu_{\mathrm{s}}$ in strange hadrons. It becomes zero crossing the $\left(T-\mu_{\mathrm{q}}\right)$-plane on the $\mu_{\mathrm{s}}=0$ curve, signifying the vanishing of the coupling in hadrons. It then becomes negative in the DQM phase because of the coupling now between the deconfined and correlated quarks and finally returns to zero as the QGP phase is approached, where quarks have bare mass and there is no coupling. The magnitude of the negative strange-quark chemical potential should be thought of in a qualitative manner in this calculation because of the approximate treatment of the dynamics in the DQM phase. A detailed, quantitative treatment of the EoS, using a 3-flavour effective Lagrangian in the Nabu-Jona-Lasinio model is underway [11].

Experimentally, central $\mathrm{S}+\mathrm{S}$ and $\mathrm{S}+\mathrm{W}$ data at $200 \mathrm{~A} \mathrm{GeV}$ of WA94/85 at CERN [12] gave the strange-particle ratios at mid-rapidity for $m_{\mathrm{T}}>1.9 \mathrm{GeV} / c$ :

$$
\begin{array}{lll}
\mathrm{S}+\mathrm{S} & \Xi^{+} / \Xi^{-}=0.54 \pm 0.06 & \bar{\Lambda} / \Lambda=0.22 \pm 0.01 \\
\mathrm{~S}+\mathrm{W} & \Xi^{+} / \Xi^{-}=0.47 \pm 0.06 & \bar{\Lambda} / \Lambda=0.196 \pm 0.011
\end{array}
$$

From these ratios we calculate

$$
\begin{gathered}
\mathrm{S}+\mathrm{S}: \quad \mu_{\mathrm{q}} / T=0.402 \pm 0.024 \quad \mu_{\mathrm{s}} / T=-0.047 \pm 0.038 \\
T=200 \pm 4 \mathrm{MeV} \quad \sqrt{\mathrm{s}} / \text { part }=9.7
\end{gathered}
$$




$$
\begin{gathered}
\mathrm{S}+\mathrm{W}: \quad \mu_{\mathrm{q}} / T=0.424 \pm 0.028 \quad \mu_{\mathrm{s}} / T=-0.033 \pm 0.043 \\
T=197 \pm 7 \mathrm{MeV} \quad \sqrt{\mathrm{s}} / \text { part }=9.1
\end{gathered}
$$

Figure 7 shows the $\mu_{\mathrm{s}} / T$ curve vs. temperature in the DQM phase for $\mu_{\mathrm{q}} / T=0.4$. The two experimental points from $\mathrm{S}+\mathrm{S}$ and $\mathrm{S}+\mathrm{W}$ interactions are put on the curve, from which their temperature is derived. Note that for the $\mathrm{S}+\mathrm{S}$ interaction, $\mathrm{R}_{\mathrm{ch}} \sim$ 0.93 and $\mathrm{R}_{\mathrm{d}} \sim 0.07$, indicating a small decrease of the hadron (quark) mass and strong qq correlation. Figure 8 shows the experimental $\mu_{\mathrm{s}} / T$ points vs. $\log (\sqrt{\mathrm{s}} /$ participant $)$, since the maximum temperature of the state varies linearly with $\log (\sqrt{\mathrm{s}} /$ participant $)$ (Fig. 1). The experimental suggestion from these two points is that, above a certain temperature the strange-quark chemical potential may become negative.

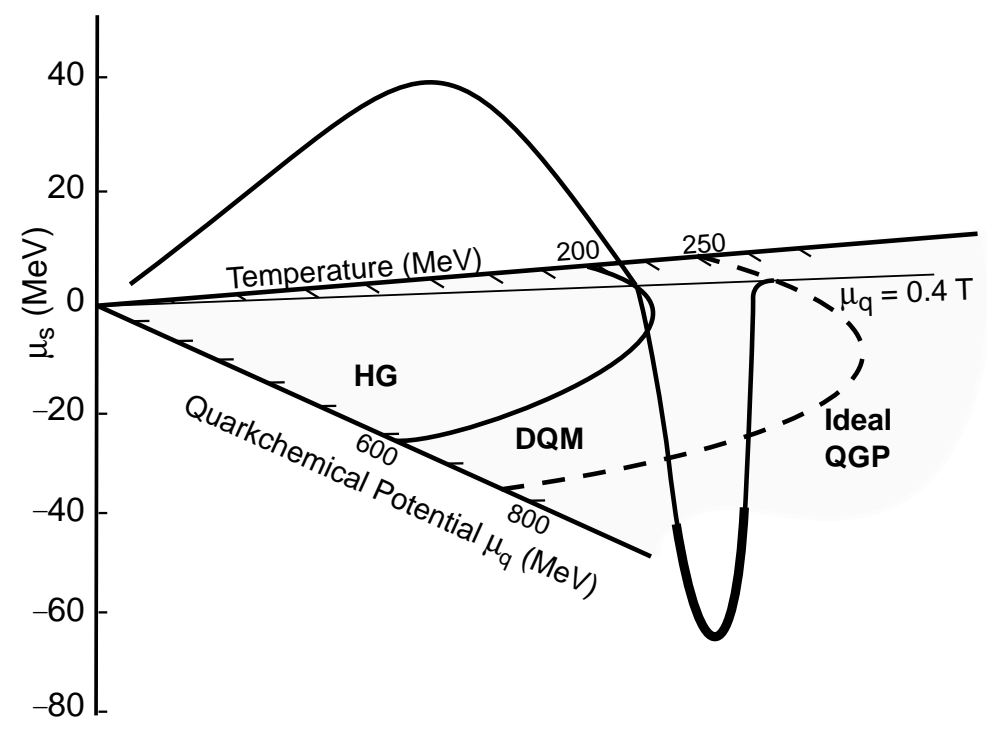

Figure 6: $\mu_{\mathrm{s}}$ vs. $T$, from Eqs. (5) and (12), shown on the vertical plane, formed by the $\mu_{\mathrm{s}}$-axis and the line $\mu_{\mathrm{q}}=0.4 T$ on the $\left(T-\mu_{\mathrm{q}}\right)$-plane.

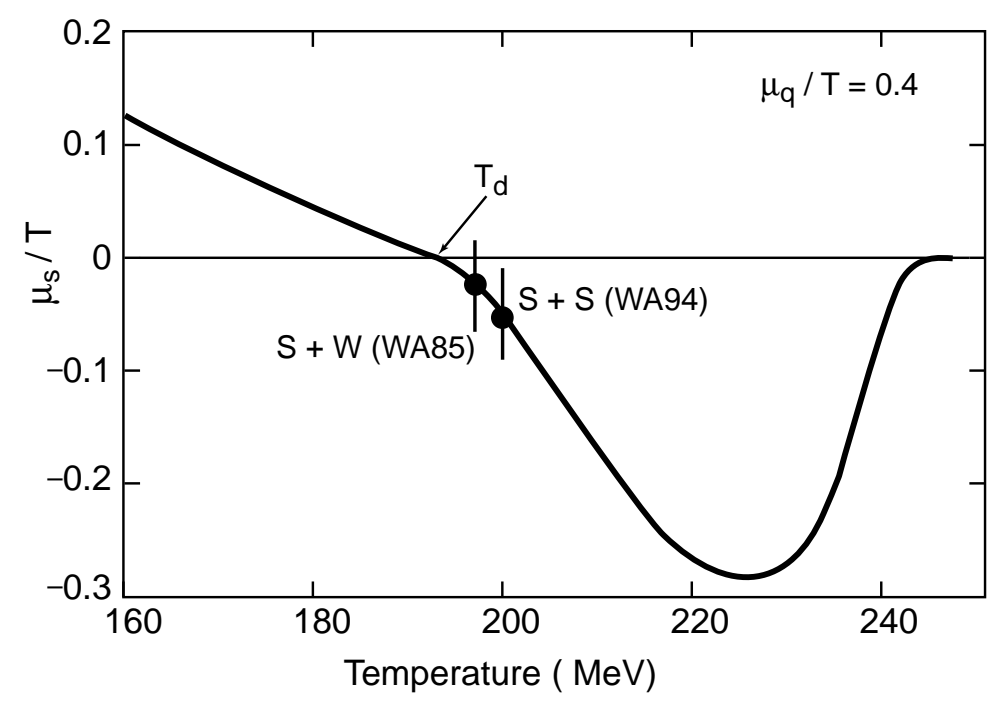

Figure 7: $\mu_{\mathrm{s}} / T$ vs. $T$ for $\mu_{\mathrm{q}}=0.4 T$ in DQM phase. Data points from $\mathrm{S}+\mathrm{S}$ and $\mathrm{S}+\mathrm{W}$ are indicated on the curve. 


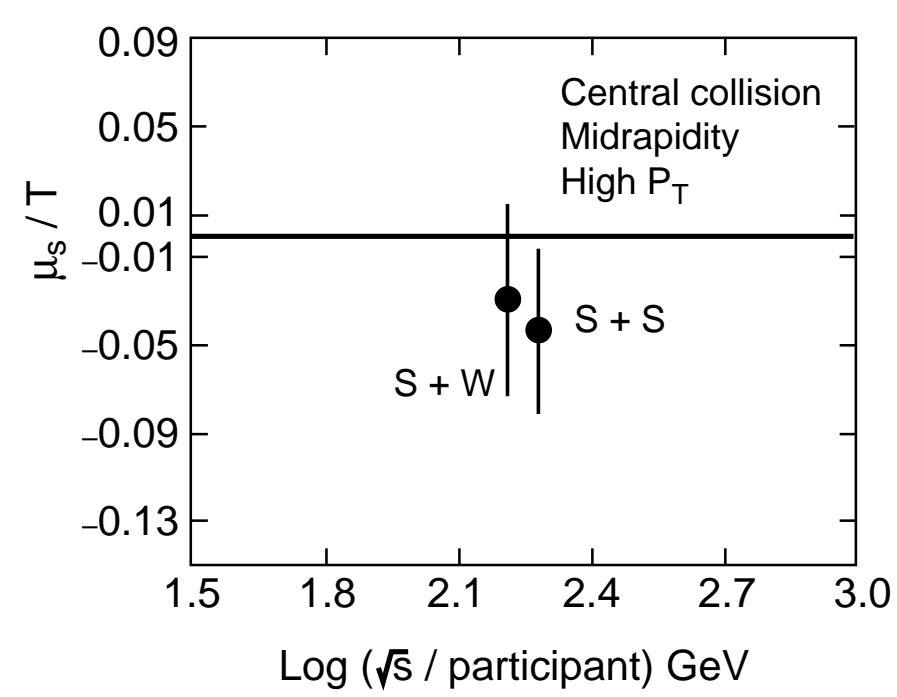

Figure 8: $\mu_{\mathrm{s}} / T$ data points from $\mathrm{S}+\mathrm{S}$ and $\mathrm{S}+\mathrm{W}$ vs. $\log (\sqrt{\mathrm{s}} /$ participant $)$.

\section{SUMMARY}

We determined the temperature of the equilibrated primordial state using the experimental $\mu_{\mathrm{q}} / T$ and $\mu_{\mathrm{s}} / T$ values obtained from strange-particle ratios. We also established the existence and determined the magnitude of the transverse-flow velocity for several nuclear interactions at AGS and SPS, thus disentangling the flow from the thermal effects. Based on our analysis, we propose the following:

- At SPS, the S-induced collisions at $200 \mathrm{~A} \mathrm{GeV}$ have reached the upper limit of hadronic physics. Some selected interactions have passed the threshold to the quark deconfinement region. Selected $\mathrm{Pb}+\mathrm{Pb}$ events (high $p_{\mathrm{T}}$, low multiplicity) at $158 \mathrm{~A} \mathrm{GeV}$ should also be situated in the DQM phase, which would be identified by the observation of large negative strange-quark chemical potential of the order of $40 \mathrm{MeV}$. For this case, $\mathrm{R}_{\mathrm{ch}} \sim 0.68$ and $\mathrm{R}_{\mathrm{d}} \sim 0.32$, indicating a substantial decrease in the hadron (quark) mass and in qq correlation of about $30 \%$.

- From the systematics, another promising interaction in terms of temperature, energy and baryon density is $\mathrm{Ag}+\mathrm{Ag}$ at $200 \mathrm{~A} \mathrm{GeV}$ (Fig. 3). Lowering the Pb-beam energy will not offer the possibility of 'new physics', since all thermodynamic quantities decrease, while the transverse-flow velocity increases (Table 1).

- $\mathrm{Au}+\mathrm{Au}$ interactions at $10.5 \mathrm{~A} \mathrm{GeV}$ are far from the deconfined state.

- At RHIC, Ni+Ni interactions at $\sqrt{\mathrm{s}}=200 \mathrm{~A}$ GeV should present an almost baryonfree mid-rapidity, concentrating the baryon number at larger rapidities and presenting the possibility for 'Centauro' events and strangelets [13]. Au+Au at $\sqrt{\mathrm{s}}<200 A$ $\mathrm{GeV}$ will produce a baryon-rich central region with high temperature. Thus RHIC will make possible the study of the decrease and disappearance of nuclear opacity at the high-energy end. 


\section{References}

[1] A.D. Panagiotou, G. Mavromanolakis, J. Tzoulis, Proc. Strangeness '95, Tucson, January 1995, AIP, New York, 1995, p. 449.

[2] A.D. Panagiotou, G. Mavromanolakis, J. Tzoulis, Phys. Rev. C53 (1996) 1353.

[3] M.N. Asprouli, A.D. Panagiotou, Phys. Rev. D51 (1995) 1086; Phys. Rev. C51 (1995) 1444.

[4] M.A. Lisa et al., EOS Collaboration.

[5] C. Kuhn et al., FOPI Collaboration.

[6] D. Krofcheck et al., Phys. Rev. C46 (1992) 1416.

[7] O.D. Chernavskaya, E.L. Feinberg, Hot hadronic matter: theory and experiment, ed. J. Letessier et al., Plenum Press, New York, 1995, and refs therein;

E. Suhonen, S. Sohlo, J. Phys. G, Nucl. Phys. 13 (1987) 1487.

[8] A.D. Panagiotou, G. Mavromanolakis, J. Tzoulis, preprint UA/NPPD-21-95 (submitted for publication).

[9] H. Satz, Proc. XXIII International Conference on High Energy Physics, Berkeley, 16-23 July, 1986, ed. S.C. Loken, World Scientific, Singapore, 1987; E. Laermann, Proc. Quark Matter '96, Heidelberg, May 1996 (to be published);

M. Jaminion, B. Van den Bossche, Z. Phys. C64 (1994) 339, and refs therein;

T. Blum, L. Kakkainen, D. Toussaint, S. Gottlieb, Phys. Rev. D51 (1995) 5153.

[10] B. Muller, Lecture Notes in Physics, Vol. 225, Springer Verlag, Berlin, 1985.

[11] A. Kapoyiannis et al., preprint in preparation.

[12] S. Abatzis et al., WA94 Collaboration, Phys. Lett. B354 (1995) 178;

D. Evans, WA85 Collaboration, Proc. Strangeness '96, Budapest, May, 1996 (to be published).

[13] A.D. Panagiotou, A. Petridis, M. Vassiliou, Phys. Rev. D45 (1992) 3134;

M.N. Asprouli, A.D. Panagiotou, E. Gladysz-Dziadus, Astropart. Phys. 2 (1994) 167. E. Gladysz-Dziadus, A.D. Panagiotou, Proc. International Symposium on Strangeness and Quark Matter, Kolymbari, Krete, September 1994, eds. G. Vassiliadis, A.D. Panagiotou, B.S. Kumar, J. Madsen, World Scientific, Singapore, 1995, p. 265. 